\title{
Viabilidad del desarrollo sostenible en un mundo insolidario: consumo energético e impacto ambiental
}

\author{
Alfredo Mingorance JimÉnez *
}

\section{ABSTRACT}

El acelerado crecimiento económico de los países desarrollados y la peligrosa imitación de este modelo por parte de los países en vías de desarrollo (PVD) suponen una seria y preocupante amenaza para los habitantes del Planeta. En el catálogo de problemas ambientales destaca el calentamiento global de la atmósfera, causado fundamentalmente por la combustión de energías fósiles y la deforestación. Por ello, se trata de disuadir a los PVD para que no subordinen su progreso a los combustibles fósiles. La consecución de este propósito depende de la solidaridad de los países ricos.

La croissance e'conomique accélérée des pays développés et la dangereuse imitation de ce modèle par les pays en voie de développement (PVD) constituent une menace inquiétante pour les habitants de la Planète. Le réchauffement global de l'atmosphère est un des plus importants problèmes de l'environnement, celui-ci est causé essentiellement par la combustion des énergies fossiles et par la déforestacion. C'est pourquoi il s'agit de dissuader les PVD afin qu'ils ne conditionnent leur progrès à ce genre de combustibles. Le succès de cet entreprise dépend absolument de la solidarité des pays riches.

The accelerated economic growth of the developped countries and the dangerous imitation of this model by the developing countries means a serious and worrying threat for the people of this planet. The global heating of the atmosphere is one of the most concerning environmental problem, which is basically caused by the burning of fossil energies and deforestation. Therefore, it is necessary to deter developing countries from using

* Doctor en Geografía. 
fossil fuels in their effort to progress. The achievement of this goal depends on the solidarity of the rich countries.

En la segunda mitad del siglo XX la crisis del desarrollo se presenta como una imprevisible amenaza que se cierne sobre el Planeta. Esta alarmante situación es el resultado de un crecimiento económico incontrolado, fomentado por un sistema capitalista de expansión absolutamente identificado con el próspero Norte que basa su esplendor en el despilfarro de los recursos naturales. Las consecuencias de este lacerante modelo no se han hecho esperar: desequilibrios ecológicos y contaminación global de la biosfera.

La responsabilidad de las opulentas sociedades occidentales insensibles a la repercusión medioambiental en su afán de crear riqueza exonera de toda acusación al débil Sur, que legítimamente pretende formar parte del selecto club del desarrollo.

Actualmente, en el umbral del siglo XXI, aparecen ineludiblemente unidas las crisis del desarrollismo y del medio ambiente. En los veinte años transcurridos desde Estocolmo (1972) hasta Río de Janeiro (1992) han ido surgiendo una serie de problemas ambientales de gran preocupación, por su magnitud planetaria, tal y como indicamos en el siguiente catálogo:

- Crecimiento de la población, fundamentalmente en el Tercer Mundo.

- Desaparición de un importante inventario, aún inconcluso, de la flora y de la fauna.

- Erosión de los suelos y los efectos de la desertización.

- Contaminación de las aguas y los residuos industriales.

- Gases invernadero y cambio climático.

- Lluvias ácidas y acidificación.

- Alteración de la capa de ozono.

* El crecimiento de la población o capacidad de carga del ecosistema global Tierra supone un mayor consumo de recursos naturales, y en muchos casos una auténtica sobreexplotación de los mismos con el consiguiente coste ecológico, que en el peor de los casos puede manifestarse localmente irreversible. La crisis de la leña en algunos países subdesarrollados es un buen ejemplo de la presión demográfica que el hombre ejerce sobre la naturaleza. Los largos desplazamientos diarios en busca de este elemental combustible condiciona extraordinariamente la dieta calorífica de millones de seres humanos que habitan las zonas más deprimidas de la Tierra, que resultan ser las de mayor crecimiento natural. 
La subalimentación y el hambre se expresan en el deterioro ambiental de extensas áreas geográficas, esquilmadas por la tala sistemática de especies vegetales que no dejan de ser vitales para la supervivencia de numerosas comunidades de pobladores del Africa Negra.

* La desaparición de una parte considerable de la riqueza florística y faunística del Planeta contribuye a la inexorable expoliación del principal legado natural, patrimonio de la humanidad, provocando un alarmante desequilibrio en aquellos ecosistemas espacialmente más afectados. La reducción de la selva amazónica, y en general los bosques tropicales sometidos a una drástica sobreexplotación en las últimas décadas, es un significativo ejemplo de la escasa sensibilidad hacia las zonas arbóreas más representativas, y por tanto irremplazables, localizadas en el mundo intertropical. En los paises tropicales la intensa deforestación por la explotación maderera y la pretendida transformación de gran parte del bosque en pasto o tierras de cultivo constituye una seria amenaza potencial, que previsiblemente puede originar cambios climáticos globales (Agenda Verde, 1993).

* La erosión de los suelos se manifiesta, en los casos de mayor intensidad erosiva, en una paulatina e incesante expansión de la desertización, sobre todo en aquellas áreas espaciales más necesitadas de recursos y que merecen una mayor protección por la acuciante presión demográfica del subdesarrollo africano. Claro ejemplo de estos irremisibles efectos de la aridez se padecen constantemente en el Sahel.

* La contaminación de las aguas es otro grave problema a escala mundial. El agua es un recurso natural escaso que debe valorarse como indispensable para la vida y para realizar la inmensa mayoría de las actividades económicas. Es irremplazable, irregular en su forma de presentarse en el tiempo y en el espacio, fácilmente vulnerable y susceptible de usos abusivos.

Dos graves peligros amenazan la disponibilidad de este recurso:

- La escasez, según la UNESCO, en quince o veinte años el agua se convertirá en el principal problema ecológico y político que deberá afrontar la población mundial. Sólo el $3 \%$ del agua del planeta es dulce, hallándose la mayor parte congelada en los casquetes polares (PNUMA, 1990).

- La poca calidad del agua, pues la propia agua de lluvia recoge múltiples contaminantes atmosféricos a lo que debemos añadir los vertidos urbanos e industriales sin depurar, fertilizantes y pesticidas empleados abusivamente en la agricultura. Por todo ello, ríos, lagos y mares se convierten en extensos depósitos contaminados, incluso los océanos no están 
libres de este riesgo. Los accidentes de los petroleros con el consiguiente vertido de crudo al mar agrava el problema. Basta recordar accidentes como el del Exxon Valdez en 1989 que arrojó más de 500.000 barriles de crudo en las costas de Alaska o los vertidos de 10 millones de barriles de petróleo al Golfo Pérsico durante la guerra del Golfo en 1991, para apreciar tamaña catástrofe ecológica (Sebastián, 1992).

Los residuos industriales consecuencia de los procesos fabriles generan anualmente 2.100 millones de toneladas de residuos sólidos, de ellos 350 millones de toneladas están considerados como peligrosos, según el Programa de las Naciones Unidas para el Medio Ambiente (PNUMA). El destino de buena parte de ellos es incierto, las aguas continentales y marítimas cargan con buena parte de ellos, depositándose en las orillas de los ríos o en las plataformas continentales, de cualquier manera no se resuelve el problema de la eliminación de los mismos.

* Los gases de invernadero y el calentamiento global de la atmósfera es el precio a pagar por las emisiones originadas en la combustión de energías fósiles. Las concentraciones de dióxido de carbono $\left(\mathrm{CO}_{2}\right)$ han aumentado un $25 \%$ en lo que va de siglo, aumentando la temperatura en la superficie de la Tierra. El $\mathrm{CO}_{2}$ es un eficaz absorbente de la radiación infrarroja de onda larga emitida por la Tierra, por lo que aprisiona el calor en la proximidad de la superficie (altas temperaturas en Venus cuya espesa atmósfera está constituída principalmente de $\mathrm{CO}_{2}$ y bajas temperaturas en Marte con una atmósfera pobre en $\mathrm{CO}_{2}$ ). Buena prueba de ello es el aumento de $\mathrm{CO}_{2}$ en la atmósfera en los últimos 50 años, pasando de 315 partes por millón (ppm) en el año 1958 a 355 ppm en el año 1990 (Houghton. Scientific American, 1991).

No cabe duda que el $\mathrm{CO}_{2}$ es el mayor contribuyente al efecto invernadero pero también otros gases, en menor medida, contribuyen a tal efecto, tales son: el metano o gas de los pantanos que se forma en la descomposición de las materias orgánicas, el óxido nitroso y los halocarburos o hidrocarburos con átomos de halógeno (fluor, cloro, bromo, yodo). Los efectos que el $\mathrm{CO}_{2}$ y el monóxido de carbono (CO) pueden tener sobre el medio ambiente en general y sobre el hombre en particular son de tal magnitud que sus consecuencias podrían incluso provocar modificaciones irreversibles en nuestro clima.

Organismos internacionales como la Organización Meteorológica Mundial (OMM) a través de un informe publicado en 1982 ha dado cuenta del peligro de aumentar las concentraciones de $\mathrm{CO}_{2}$ si el consumo de energía fósil continuaba creciendo en los próximos años. La mayoría de las proyecciones coinciden en señalar que el contenido de $\mathrm{CO}_{2}$ se duplicará durante el siglo $\mathrm{XXI}$, con el consiguiente aumento de temperatura a 
escala global y el riesgo de fusión parcial de las capas de hielo del Atlántico Norte y los casquetes polares subiendo el nivel del mar (Organización Metereológica Mundial, 1982).

En 1990, según el PNUMA, por consumos energéticos se emitieron a la atmósfera 5.750 millones de toneladas de $\mathrm{CO}_{2}, 177$ millones de toneladas de $\mathrm{CO}, 99$ millones de toneladas de $\mathrm{SO}_{2}$ (dióxido de azufre), 68 millones de toneladas de $\mathrm{NO}_{x}$ (óxidos de nitrógeno) y 57 millones de toneladas de partículas en suspensión.

Ahora bien, la corresponsabilidad en las emisiones de $\mathrm{CO}_{2}$ varía considerablemente entre los países ricos desarrollados y los países pobres subdesarrollados. En el año 1991 EE.UU. emitía a la atmósfera 5,8 toneladas por habitante y Bangladesh 0,1 toneladas por habitante.

La participación porcentual de las distintas fuentes y gases en el efecto invernadero, y por consiguiente, en el calentamiento global resultante es la siguiente, según el cuadro adjunto:

\section{PARTICIPACIÓN DE LAS DISTINTAS FUENTES Y GASES EN EL EFECTO INVERNADERO}

(Porcentaje)

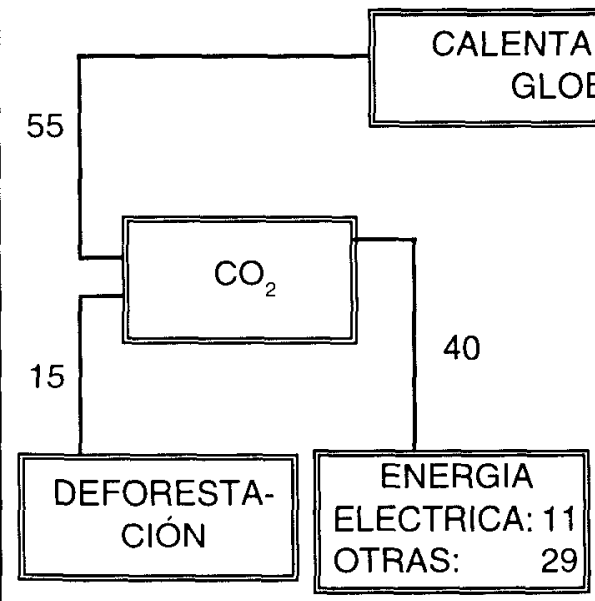

Fuente: Atomic Energy Agency (Reino Unido)

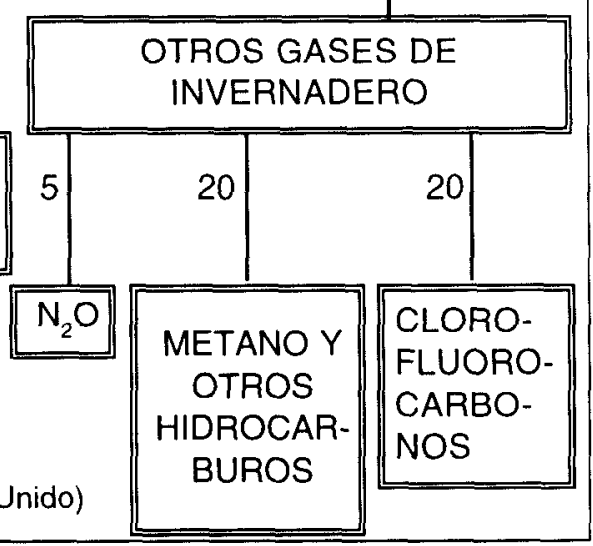


En cuanto a las actividades humanas que pueden producir calentamiento global aparecen reflejadas en el siguiente diagrama:

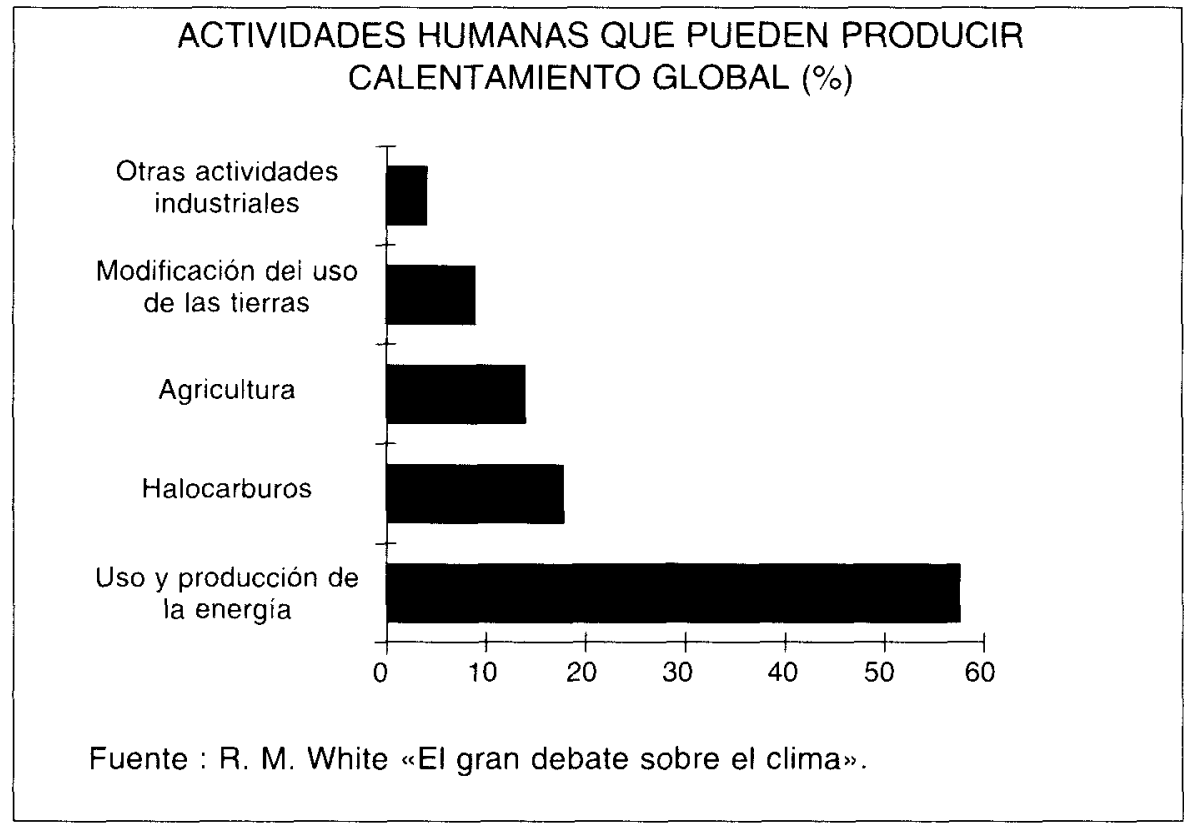

No está claro la cantidad precisa de calentamiento ni la distribución regional de cambio climático que puede esperarse en la Tierra del aumento en la concentración de $\mathrm{CO}_{2}$. Sin embargo, un hecho es cierto la Tierra ha aumentado $0,5^{\circ} \mathrm{C}$ la temperatura a lo largo de este siglo. El calentamiento de las capas inferiores de la atmósfera por el "efecto invernadero" podría alterar los procesos hidrológicos y la distribución local de los regímenes de lluvias, aunque aún no se conocen bien sus efectos a escala regional.

Otra preocupación en el posible cambio climático, por la actividad humana, es la modificación de los suelos por efecto de la deforestación, la sobreexplotación de los pastos y la desertización, así como la tala de árboles. La crisis de la leña o la utilización de la biomasa como principal fuente de energía para usos domésticos afecta a más de 2.500 millones de personas en el Tercer Mundo (Jiménez Herrero, 1992). La leña supone el $35 \%$ del consumo energético en los paises en vías de desarrollo (PVD), y en algunos paises africanos como Tanzania alcanza el 90\%. Estas actividades pueden aumentar el albedo (reflejo de la luz solar) de la superficie terrestre, por lo que la luz solar absorbida es menor. La Tierra se enfría provocando alteraciones en las capas inferiores de la atmósfera, presencia 
de aire frío sobre la superficie terrestre y aire caliente en las capas superiores reduciendo la actividad convectiva de la atmósfera, lo cual supone la inhibición de nubes y precipitaciones.

Es incuestionable que el $\mathrm{CO}_{2}$ en la atmósfera aumenta por la combustión de energías fósiles y la tala de bosques. $\mathrm{El} \mathrm{CO}_{2}$ produce un efecto invernadero, del mismo modo que el vidrio de un invernadero permite el paso de la luz solar pero evita que escape el calor, al impedir que el aire caliente del interior del invernadero se mezcle con el aire del exterior, de manera análoga, el $\mathrm{CO}_{2}$ y otros gases de invernadero (metano, óxido nitroso y los halocarburos) muestran una relativa transparencia a la luz solar pero aprisionan de forma eficaz el calor al absorber la radiación infrarroja de mayor longitud de onda emitida por la Tierra. El $\mathrm{CO}_{2}$ altera el balance de calor de la Tierra al actuar como pantalla e impedir la dispersión hacia el espacio de la radiación infrarroja, de mayor longitud de onda, que irradia la superficie de la Tierra.

Con las proporciones actuales de combustibles y la velocidad del crecimiento de su consumo, la mayor parte del petróleo y del gas natural estará agotada hacia el año 2050, mientras que quedará el 90-95\% de carbón explotable concentrado en tres países: la extinta Urión Soviética, China y EE.UU., por lo que estos tres paises jugarán un papel decisivo en cualquier futuro incremento en la cantidad de $\mathrm{CO}_{2}$ atmosférico.

En cualquier caso, hoy resulta imposible interpretar exactamente el innegable calentamiento a escala global de los últimos cien años. Faltan datos históricos y sólo disponemos de modelos de ordenador que predicen que los gases capaces de retener calor que se han lanzado a la atmósferá en los últimos 200 años harán subir la temperatura media mundial entre $1^{\circ}$ y $4^{\circ} \mathrm{C}$ en los próximos 50-70 años. Pero estos modelos informáticos son simplificaciones incapaces de reproducir los complicados procesos que se desarrollan en la atmósfera y en los océanos, por lo que no pueden demostrar que las emisiones de gases de invernadero vayan a alterar apreciablemente el clima terrestre. Los modelos matemáticos coinciden en señalar que un aumento del $\mathrm{CO}_{2}$ conduciría a un calentamiento gradual que dependería de la tasa de uso global de la energía fósil, pero el aumento de temperatura que se produciria debido a la duplicación del $\mathrm{CO}_{2}$ varía bastante de un modelo a otro. No debemos olvidar que los modelos matemáticos informáticos son solo aproximaciones que intentan simular los procesos que rigen el comportamiento atmosférico, solamente como pronóstico y nunca como interpretación de la complejidad de los procesos fisicoquímicos en el intercambio de $\mathrm{CO}_{2}$ entre la atmósfera y la Tierra (Houghton. Scientific American, 1991). 
Más allá de estas observaciones sobre la tendencia hacia el calentamiento global, el mayor compromiso humano es impedir, o al menos frenar, las dos fuentes principales de emisiones de $\mathrm{CO}_{2}$ : la combustión de energías fósiles y la deforestación. Cada año desprendemos a la atmósfera unos 5.600 millones de toneladas de carbono, aportando los países industrializados el $75 \%$ de estas emisiones. España contribuye con más de 50 millones de Tn de carbono (Forum Atómico Español, 1993).

Los PVD si siguen el mismo modelo de desarrollo económico incrementarán su contribución potencial, por lo que es necesario encontrar modelos alternativos que rebajen la dependencia de los combustibles fósiles. Por otra parte, la deforestación sobre todo en los trópicos ha aumentado peligrosamente en la última década. La repoblación forestal ayudaría a estabilizar la composición de la atmósfera. El caso de Brasil destinando grandes extensiones de tierras fértiles para producir alcohol de automoción es al menos criticable, y por tanto, no aconsejable, porque la recuperación de las masas boscosas requiere una mayor temporalidad que la que se necesita para la tala abusiva (Jiménez Herrero, 1992).

Es absolutamente necesario que los PVD consigan tecnología del rico Norte para apostar por la diversificación energética, lo que significaría la reducción de $\mathrm{CO}_{2}$ y del calentamiento global terrestre. La apuesta más correcta, hoy por hoy, no es otra que la del abandono progresivo del consumo de energías fósiles y la potenciación de las energías renovables, que no alternativas, como energías sustitutorias o de cambio. Su contribución al balance energético mundial sigue siendo reducida, apenas superan el umbral de la competitividad aunque en los últimos años han alcanzado un considerable desarrollo tecnológico los aerogeneradores instalados en los parques eólicos y la energía solar fotovoltaica. Ambas energias, la eólica y la solar, también presentan un cierto impacto ambiental. En cualquier caso, la potencia instalada apenas supera algunos megavatios que resultan simbólicos frente a los más de $500 \mathrm{MW}$ de las centrales convencionales tanto térmicas como nucleares, alcanzando estas últimas hasta algo más de $1000 \mathrm{MW}$ de potencia instalada. También debe tener cabida la energía nuclear de fisión siempre que el problema de los residuos radiactivos tenga un tratamiento satisfactorio, principalmente los residuos de alta actividad (RAA) en almacenamientos definitivos (depósitos geológicos profundos graníticos, salinos o yesíferos). La presión social no debe dejarse influenciar por un falso ecologismo sin sustentación científica, ya que lejos de aportar soluciones a las emisiones de $\mathrm{CO}_{2}$ y otros gases de invernadero a la atmósfera, estas actitudes contribuyen a agravar el problema del cambio climático, del cual todavía ignoramos las consecuencias derivadas de tal efecto. Se deben favorecer posturas que 
se inclinen por las energías limpias y no contaminantes y desoir argumentos demagógicos amparados en la ecología incapaces de responder adecuadamente a las necesidades energéticas actuales.

* Las lluvias ácidas aparecen como otra preocupación medioambiental del mundo industrializado irrespetuoso con la naturaleza. Se deben al azufre y al nitrógeno liberados en la atmósfera que al combinarse con el agua se transforman en moléculas de ácidos de azufre (sulfúrico) y nitrógeno (nítrico), en un $70 \%$ y $30 \%$ respectivamente.

Los combustibles fósiles son los causantes de las lluvias ácidas, sobre todo el carbón de elevado contenido de azufre. En 1982 la Conferencia Mundial sobre la Acidificación del Medio presentó un dato revelador del impacto ambiental: "anualmente se emiten en el mundo unos 100 millones de toneladas de azufre". Las lluvias ácidas empiezan a ser un problema fundamental, los lagos y los bosques empiezan a morir por efecto de la acidificación del suelo y de las aguas superficiales y subterráneas (precipitación ácida $\mathrm{pH}$ inferior a 5,6). Las precipitaciones ácidas pueden producirse tanto cerca de los focos de contaminación como lejos de ellos. Las precipitaciones convectivas se originan a partir de corrientes verticales ascendentes generadas por el calentamiento solar del suelo, tienen carácter local, y abarcan menos de 100 kilómetros y poseen una corta duración temporal menos de una hora. Las precipitaciones unidas a fenómenos de transporte transfronterizo se manifiestan bien a escala sinóptica, abarcan hasta 1.000 kilómetros y duran entre 10 y 100 horas; o a escala planetaria, cuando abarcan unos 10.000 kilómetros y duran unas 100 horas. Las chimeneas de las centrales térmicas han elevado su altura por encima de los 180 metros favoreciendo la dispersión de las emisiones y el transporte transfronterizo, algunas partículas de chimeneas altas pueden recorrer hasta 2.000 kilómetros en solo dos o tres dias.

El impacto ambiental de las lluvias ácidas es otro de los graves problemas que padecen las sociedades industrializadas que abusan del consumo de energías fósiles, principalmente carbón, tal y como ocurre en Europa Central y Oriental donde los efectos devastadores se han dejado sentir en la reducción de la cubierta forestal. Ha desaparecido la mitad de los bosques de Alemania y el $75 \%$ de Checoslovaquia (Sebastián, 1992). Resulta especialmente significativo en este sentido la emisión de más de 39 millones de toneladas de óxidos de azufre por los países de la OCDE en el año 1989 (Forum Atómico Español, 1993).

* Otra alteración medioambiental es debida a los daños causados en la estratosfera entre los 14 y los 50 kilométros de altitud donde se concentra el $90 \%$ del ozono atmosférico. A esta altitud se ha detectado un alarmante 
descenso de la capa de ozono, fenómeno conocido como "agujero de ozono". Esta capa ozonosférica absorbe la mayor parte de la radiación solar ultravioleta B (UVB) evitando que llegue a la superficie terrestre con toda su intensidad. Hasta hoy, la alteración se manifiesta en una disminución del ozono $\left(\mathrm{O}_{3}\right)$ en las zonas polares donde el aire inestable tiene mayor cantidad de ozono que el aire tropical estable originándose un fenómeno climatológico conocido como «vórtice polar» en el que los vientos huracanados impiden el intercambio de masas de aire entre el interior y el exterior del vórtice y aislar dentro de él masas de aire muy frío con contaminantes, entre ellos el cloro activado que tarda entre 10 y 15 años en activarse, $y$ destruyen el ozono en un proceso muy rápido al no existir intercambio con masas de aire que traigan ozono de otras partes de la Tierra (White. Scientific American, 1991).

En 1987, el Protocolo de Montreal acordó reducir al 50\% los hidrocarburos clorofluorados (C.F.C.s) o freones empleados como refrigerantes, extintores de espuma, disolventes industriales y como propulsores de aerosoles o sprays para el año 1999. Los C.F.C.s se descomponen en la estratosfera produciendo átomos de cloro que destruyen el ozono. En 1990, el Protocolo de Londres ante la alarmante destrucción de la capa de ozono en la Antártida decidió aumentar al 100\% la reducción, es decir, eliminar los C.F.C.s para ese año. Pero ante la alarmante destrucción de la capa de ozono el PNUMA ha propuesto su total eliminación para 1995 (Agenda Verde, 1993). Muchos países no han firmado este nuevo protocolo, entre ellos España.

Analizado el catálogo de problemas ambientales evidenciamos un conjunto de peligros que amenazan el desarrollo sostenible en Gaia. Debemos renunciar a un mal uso de la energía y comprometernos a mejorar los usos de la energía para obtener un mayor rendimiento de la misma y reducir la dependencia de los combustibles fósiles. La eficiencia energética (menor consumo de energía por unidad de volumen) disminuye la contaminación por la reducción de las emisiones de óxidos de azufre y nitrógeno, la lluvia ácida y la liberación de otras toxinas. La energía que menos contamina es la que no se produce ni se consume, la mejor opción es, por consiguiente, el ahorro energético. Hay que fomentar la cogeneración, producción y aprovechamiento simultáneo de energía térmica y electricidad. Los propósitos son conseguir productos menos intensivos en energía, menos contaminantes, con más valor añadido y generadores de empleo.

La energía nuclear de fisión contribuye a la reducción de contaminantes e impide la emisión de gases de invernadero, pero su utilización lleva aparejado el volumen de residuos radiactivos de alta, media y baja actividad 
(RAA, RMA, RBA). Habrá que esperar 40 ó 50 años para que la energía de fusión se convierta en la energía del futuro con mínimas repercusiones ambientales, al desaparecer el problema de los residuos radiactivos. Mientras tanto, las energias renovables no pueden presentarse como una alternativa resolutoria, para el año 2.010 sólo representarán el equivalente al $15 \%$ de la demanda de energía primaria convencional en la Unión Europea, según el Plan de Acción para las Fuentes de Energias Renovables en Europa aprobado en la Declaración de Madrid en marzo de 1994, quedando por resolver el otro $85 \%$ de producción de energia primaria (Conferencia de Madrid, 1994).

Ante este panorama los planes energéticos nacionales deben basarse en la diversificación energética con una decidida potenciación de aquellas fuentes de energía menos contaminantes y con menor impacto ambiental.

Es ineludible detener la pérdida de bosque para mantener la diversidad genética del Planeta, reducir la erosión, estabilizar climas locales y regionales, limpiar el aire y el agua, en definitiva, guardar el legado de la naturaleza que permita vivir a las generaciones venideras.

El camino hacia una sociedad global económicamente viable, socialmente justa y ecológicamente sostenida conduce a una incesante búsqueda para satisfacer las necesidades de las generaciones actuales sin comprometer la capacidad de las generaciones futuras. Esta apuesta será alcanzable si se consigue el objetivo permanente de mantener el complejo equilibrio vital establecido desde hace millones de años.

Países ricos (Centro) y mundo subdesarrollado o países en vías de desárrollo, PVD (Periferia) no pueden escapar de la mundialización de los problemas económicos y ambientales. El desarrollo económico ha originado una ecocrisis mundial donde los costes ambientales y ecológicos están desproporcional e injustamente repartidos para las poblaciones subdesarrolladas o PVD. Se debe poner freno, al menos, tratando de impedir la destrucción de los recursos naturales, la deforestación no tiene respuesta inmediata en la reforestación, la repoblación vegetal es un ciclo natural temporalmente lento. Oposición frontal a la sobreexplotación de los ecosistemas, a la destrucción de suelos y a la degradación de los bosques tropicales con una salvaje deforestación que está causando un desastre ecológico difícilmente reparable.

Un desarrollo económico respetuoso con el medio obliga a aceptar una nueva terminologia económico-ecológica tal y como apunta $\mathrm{H}$. Daly al hablar de producto nacional neto social sostenible: «máxima cantidad que puede ser consumida en un periodo de tiempo sin reducir la capacidad 
de consumo posible en un periodo futuro", indicador válido para el bienestar económico sostenible (Jiménez Herrero, 1992).

La Cumbre de la Tierra celebrada en junio de 1992 en Río de Janeiro, dándose cita los países ricos y los países pobres, dejó bien sentada la corresponsabilidad en los problemas medioambientales. Lícitamente el derecho al crecimiento económico de los PVD no debe obligarles a renunciar a tecnologías nocivas (C.F.C.s) para el medio ambiente en tanto que los países económicamente poderosos no les proporcionen tecnologías compatibles con la protección de los ecosistemas naturales. Los países ricos carecen de legitimidad para exigir la renuncia de los PVD a usar determinadas tecnologías agresivas para con la naturaleza cuando el mundo desarrollado e industrializado a lo largo de este siglo ha tenido un comportamiento irrespetuoso, despreciando la conservación de los entornos naturales, lanzando a la atmósfera todo tipo de emisiones contaminantes y sustancias tóxicas. No ha existido conciencia política para atajar las reiteradas hostilidades hacia la biosfera. Éticamente no se les puede pedir a los PVD que renuncien a procesos de industrialización obligándoles a cumplir responsabilidades ambientales. Un clamor unánime debe manifestarse para liberar a estos países de las tres peores "contaminaciones" que padecen: el hambre, la miseria y la marginación. Y la implicación de las "élites tradicionales" de estos países, como ocurre en América Latina, no debe diluirse en un vago voluntarismo solapado por la falta de un compromiso real (Sebastián, 1992).

El problema de la toma de conciencia y de las actuaciones para detener la degradación paulatina de la Naturaleza sencillamente debe plantearse en términos consecuentes, cargar con los costos en la misma medida que se reciben beneficios, por lo tanto, los países más beneficiados deben soportar las mayores cargas simplemente por corresponsabilidad. Para impedir el fracaso de los compromisos de Río los países pobres necesitan ayudas de los países ricos para asumir la corresponsabilidad de Río sobre cambio climático y biodiversidad. La Cumbre de la Tierra significó aceptar unánimemente que los impactos ambientales locales tienen repercusiones globales y que ningún gobierno bajo el amparo de la soberanía debe defender cualquier tecnología perjudicial para el medio ambiente. Los problemas ambientales han dejado de ser locales o regionales para convertirse en problemas transfronterizos que preocupan y resuelven los organismos internacionales competentes con actuaciones inmediatas, incluso con métodos expeditivos, para frenar e impedir cualquier agresión medioambiental. La cuestión ecológica es el banderín de enganche en una hipotética intervención internacional contra el agresor o infractor, justificando de este modo el posible imperialismo ecológico que pudiera existir 
en defensa y protección del Planeta. El delito ecológico atenta contra el patrimonio de la humanidad y requiere una respuesta firme de rechazo y el empleo de medidas coercitivas. El "ecodesarrollo" es un fin y los medios para conseguirlo deben estar al alcance del Tercer Mundo, de no ser así todo quedará en una decepcionante aspiración humana fruto de la colectiva irresponsabilidad de los moradores terrestres.

\section{BIBLIOGRAFIA COMPLEMENTARIA}

- Agenda Verde (1993). Documentación Verde. Madrid. TransEdit, S.L.

- Conferencia de Madrid (1994): "Un Plan de Acción para las Fuentes de Energias Renovables en Europa". Madrid 16-18 marzo 1994.

- Forum Atómico Español, FAE (1993): “Energía 93". Madrid.

- HOUGHTON, RICHARD A. y WOODWELL, GEORGE M. (1991): CCambio climático global". El Clima. Libros de Investigación y Ciencia. Scientific American. Barcelona.

- Informe de la Organización Metereológica Mundial, OMM (1982)

- JIMENEZ HERRERO, LUIS M. (1992): "Medio Ambiente y Desarrollo Alternativo". Madrid, lepala.

- Programa de las Naciones Unidas para el Medio Ambiente, PNUMA (1990).

- SEBASTIÁN, LUIS DE (1992): "Mundo Rico, Mundo Pobre. Pobreza y Solidaridad en el mundo de hoy". Santander, Editorial Sal Terrae.

- WHITE, ROBERT M. (1991): "El gran debate sobre el clima". El Clima. Libros de Investigación y Ciencia. Scientific American. Barcelona. 